\title{
Restoration of polr1c in Early Embryogenesis Rescues the Type 3 Treacher Collins Syndrome Facial Malformation Phenotype in Zebrafish
}

\author{
Ernest Man Lok Kwong, ${ }^{*}$ Jeff Cheuk Hin Ho, ${ }^{*}$ Marco Chi Chung Lau, ${ }^{*}$ May-Su You, ${ }^{\dagger}$ Yun-Jin Jiang, ${ }^{\dagger}$ and William Ka Fai Tse
}

From the Department of Biology, ${ }^{*}$ Hong Kong Baptist University, Kowloon Tong, Hong Kong; the Institute of Molecular and Genomic Medicine, ${ }^{\dagger}$ National Health Research Institutes, Miaoli, Taiwan; and the Faculty of Agriculture, ${ }^{\ddagger}$ Kyushu University, Fukuoka, Japan

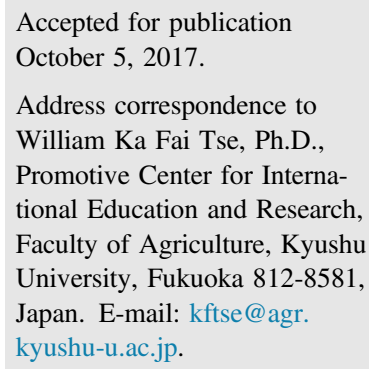

\begin{abstract}
Treacher Collins syndrome (TCS) is a rare congenital birth disorder ( 1 in 50,000 live births) characterized by severe craniofacial defects. Recently, the authors' group unfolded the pathogenesis of polr1c Type 3 TCS by using the zebrafish model. Facial development depends on the neural crest cells, in which polr1c plays a role in regulating their expression. In this study, the authors aimed to identify the functional time window of polr1c in TCS by the use of photo-morpholino to restore the polr1c expression at different time points. Results suggested that the restoration of polr $1 \mathrm{c}$ at 8 hours after fertilization could rescue the TCS facial malformation phenotype by correcting the neural crest cell expression, reducing the cell death, and normalizing the $p 53$ mRNA expression level in the rescued morphants. However, such recovery could not be reproduced if the polr1 $\mathrm{C}$ is restored after 30 hours after fertilization. (Am J Pathol 2018, 188: 336-342; https://doi.org/10.1016/j.ajpath.2017.10.004)
\end{abstract}

Treacher Collins syndrome (TCS) [Online Mendelian Inheritance in Man (OMIM) no. 154500] is an autosomaldominant mandibulofacial dysostosis that occurs in 1 out of 50,000 live births. ${ }^{1}$ It is a rare congenital birth disorder characterized by severe craniofacial defects. ${ }^{2}$ TCS affects the proper formation of facial structure, which leads to profound facial dysmorphism. ${ }^{3}$ Various facial phenotypes have been reported, including downward slant of palpebral fissure secondary to hypoplasia of the lateral orbit and cleft palate. ${ }^{4}$ Detailed human clinical description can be referred to clinical reviews. ${ }^{5}$

TCS can be classified into three types based on the origin of mutation: TCS1 has the indicated mutation in TCOF1 [Type 1 TCS (TCS-1); OMIM no. 154500], whereas TCS2 has a mutation in POLRID (TCS-2; OMIM no. 613717). TCS with a POLRIC mutation is classified as Type 3 TCS (TCS-3; OMIM no. 248390). Although most cases of TCS are attributed to mutations in TCOF1, mutations in POLRIC and POLRID account for $9 \%$ of all non-TCOF1-related TCS cases. ${ }^{6,7}$ The disease mechanism is closely related to an increased expression of $p 53$, leading to cell cycle arrest and apoptosis. 8,9 Recently, our group has uncovered the pathogenesis of the POLRIC-related TCS-3, and suggested the genetic suppression of $p 53$ may rescue the disease. ${ }^{10}$ In this report, the functional time window of the polrlc is identified, and more importantly, it is demonstrated that the restoration of polrlc in the early stage of development may partially rescue the TCS facial phenotype via restoring the neural crest cell (NCC) expression, reducing the cell death population, and guiding the $p 53$ pathway back to normal. Collectively, this is the first study to suggest the polrlc developmental functional time window in zebrafish, which provides new insight on reducing the treatment time under pharmacotherapy.

\footnotetext{
Supported by the Japan Society for the Promotion of Science Bilateral Open Partnership Joint Research Project AJ179064 (W.K.F.T.), National Health Research Institutes, Taiwan, grants MG-105-PP-12 and MG-106PP-11, and the Ministry of Science and Technology, Taiwan, grants MOST 105-2311-B-400-001, MOST 106-2319-B-400-002, and MOST 106-2311-B-400-003-MY3 (Y.-J.J.).

E.M.L.K. and J.C.H.H. contributed equally to this work.

Disclosures: None declared.
} 


\section{Materials and Methods}

\section{Fish Strains and Maintenance}

The zebrafish strains used in this study were the $A B$ wildtype and $T g(f i l a: G F P)$ lines that were obtained from the Zebrafish International Resource Center (Eugene, OR). They were raised and staged as previously described. ${ }^{11}$ All experimental procedures on zebrafish embryos were approved by the Animal Experimental Committee, Hong Kong Baptist University.

\section{MO Sequence Site Selection, Design, Injection, and Cleavage}

The design of the morpholinos (MOs) was described previously, ${ }^{12}$ and they were generated by Gene Tools, LLC (Philomath, OR). For the polrlc splicing Photo-morpholino (PMO), oligo targeting intron1 and exon2 (5'TGGACCTGTAAAPATTAGATACAGT-3 ${ }^{\prime}$ ) was generated. The cleavage site is underlined. PMO was resuspended in distilled water and stored at $-20^{\circ} \mathrm{C}$. Two picomole of polrlc PMO was injected into 1- or 2-cell stage embryos. Embryos from four different pairs of fish were used for each PMO injection, and the injections were administered on multiple days. More than 200 embryos from four pairs of zebrafish were used for injection per day. For the PMO cleavage, a Gene Tools ultraviolet (UV) lightbox was used as per the manufacturer's protocol. Briefly, the control or PMO embryos (10 embryos each time) at selected developmental stages were put into the flat-bottomed tube provided, followed by 300 seconds of narrow-bandwidth $365-\mathrm{nm}$ light. After exposure, embryos were placed back in the Petri dish with E3 medium.

\section{RNA Extraction, Reverse Transcription, and Real-Time Quantitative PCR}

Two and 5 days post-fertilization (dpf) larvae were collected. Ten larvae were pooled as 1 sample, and 12 samples with duplication were used for each experimental set.

Their total RNA was extracted by using TRIzol (Thermo Fisher Scientific, Waltham, MA). Purified RNA with an A260/A280 ratio of 1.8 to 2.0 was used. Briefly, $0.5 \mu \mathrm{g}$ of total extracted RNA was reversely transcribed (ViLO; Thermo Fisher Scientific). Real-time PCR was conducted with the Applied Biosystems 7500 real-time PCR detection system using KAPA SYBR Green Supermix (Applied Biosystems, Foster City, CA). Beta-actin was used for normalization, and the relative expression ratio was calculated according to the described method. ${ }^{13}$ The sequences of the $p 53$ primer were forward: $5^{\prime}$-TTAAGTGATGTGGTGCCTGCCT-3'; reverse: 5'-AGCTTCTTTCCCTGTTTGGGCT- $3^{\prime}$.
Alcian Blue Cartilage Staining and Facial Malformation Analysis

Five-dpf larvae were collected and fixed with $4 \%$ paraformaldehyde, and then followed by the general staining protocol as previously described. ${ }^{14}$ Quantification of the ethmoid plate morphology was used to represent the facial phenotype at 5 dpf. Samples were first randomly selected, and their whole-mount Alcian blue staining was confirmed by two individual researchers before dissecting the ethmoid plate for measurement. Length, width, length/width ratio, and relative area of the plate were measured. ImageJ software version $1.50(\mathrm{NIH}$, Bethesda, MD) was used.

\section{Whole-Mount in Situ Hybridization, Acridine Orange Staining, and Imaging}

One-dpf embryos were collected and fixed with $4 \%$ paraformaldehyde. Single in situ hybridization of $d l x 2 a$ was performed as previously described. ${ }^{15}$ For the Acridine orange staining, 2-dpf embryos were bathed in $10 \mu \mathrm{g} / \mathrm{mL}$ Acridine orange (Sigma-Aldrich, St. Louis, MO) in the dark for 1 hour, followed by replacing the Acridine orange solution with E3 medium before imaging. Images were captured by a Leica fluorescence microscope (Leica, Wetzlar, Germany) and quantified by ImageJ software as previously described. ${ }^{10,16}$

\section{Results}

Restoration of polr1c at Early Developmental Stage May Rescue the Facial Phenotype

To unfold the critical developmental time window of the polrlc, the modified polrlc $\mathrm{PMO}$ with the reported sequence was used. ${ }^{10}$ The modification was made for the MO cleavage by the specific $365-\mathrm{nm}$ UV wavelength, allowing the protein translation process. The use of PMO to "turn on" the gene has been reported in different models. ${ }^{17-19}$

To identify the functional time window of polrlc, different time points $[8,16,24,30$, and 48 hours post-fertilization (hpf)] were screened for polrlc restoration. The data are reported in Supplemental Figure S1. The cleavage and the Alcian blue staining were performed at different time points. Results showed that the ethmoid plate structure was malformed in morphants that were exposed to UV light at 16, 24, 30, and 48 hpf (Figure 1and Supplemental Figure S1). Morphants exposed to UV light at 16 and $24 \mathrm{hpf}$ exhibited a wide range of ethmoid plate structures (in terms of length, width, and relative area), which indicated the restoration of polrlc at such time points was unable to rescue the facial structure at a high successful rate. On the other hand, the morphants exposed to UV light at 8 and 30 hpf may result in a more consolidate phenotype. Two representative time points were chosen for further investigation. The cleavage was performed at 8 and 30 hpf. The modified PMO mimicked the facial 

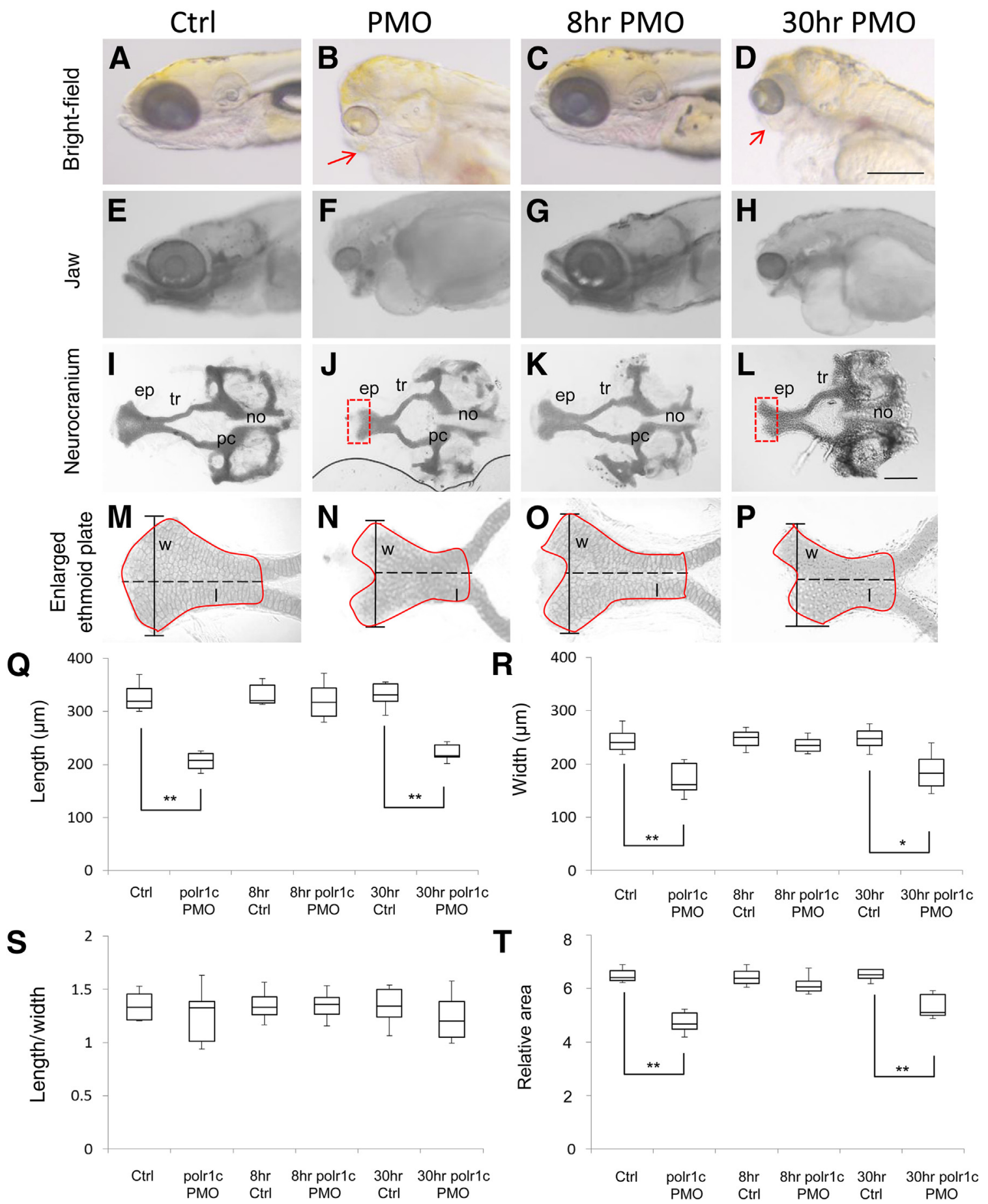

Figure 1 Restoration of polr1c via PM0 in the early developmental stage may rescue TCS facial phenotypes at 5 dpf. A-D: polr1c P-morphants exhibit a rounded head, smaller eyes, and jaw agenesis at $5 \mathrm{dpf}$. Arrows indicates the malformation of the lower jaw in morphants (B and $\mathbf{D})$. The phenotype can be rescued by polr1c restoration at 8 hours (C), but not at 30 hours (D). E-H: Alcian blue staining clearly demonstrates the loss of facial cartilage in P-morphants (F) and P-morphants that were exposed to 365-nm UV light at 30 hours (H). On the other hand, the P-morphants that were exposed to UV light at 8 hours show a rescued facial phenotype (G). I-L: Flat mount of the neurocranium shows the rudimentary ethmoid plate structure (red dashed boxes) in P-morphants (J) and P-morphants that were exposed to UV light at 30 hours (L). M-P: Enlarged view of the ethmoid plate. The plate is outlined in red. The P-morphants (L) and P-morphants that were exposed to the UV light at 30 hours $(\mathbf{P})$ have a shorter and smaller ethmoid plate. Dashed lines indicate the length of the plate; solid lines, the width $(w)$. The malformation phenotypes shown are the representative figures of severe cases. Anterior is to the left in all views. E-P: Images were recolored grey for better contrast. Q-T: Quantified data of the ethmoid plate structure. The length (Q), width (R), and the relative area $(\mathbf{T})$ of the ethmoid plate are greatly reduced in the P-morphants and P-morphants that were exposed to the UV light at 30 hours. However, there is no significant difference in the P-morphants that were exposed to the UV light at 8 hours. In addition, there is no significant change in length-to-width ratio among all of the samples (S). $n=35(\mathbf{Q}-\mathbf{T}) .{ }^{*} P<0.05,{ }^{*} P<<0.01$. Scale bars: $500 \mu \mathrm{m}(\mathbf{A}-\mathbf{H}) ; 200 \mu \mathrm{m}(\mathbf{I}-\mathbf{L})$. Original magnification, $\times 100(\mathbf{M}-\mathbf{P})$. Ctrl, control; ep, ethmoid plate; no, notochord; pc, parachordal; tr, trabecula. 

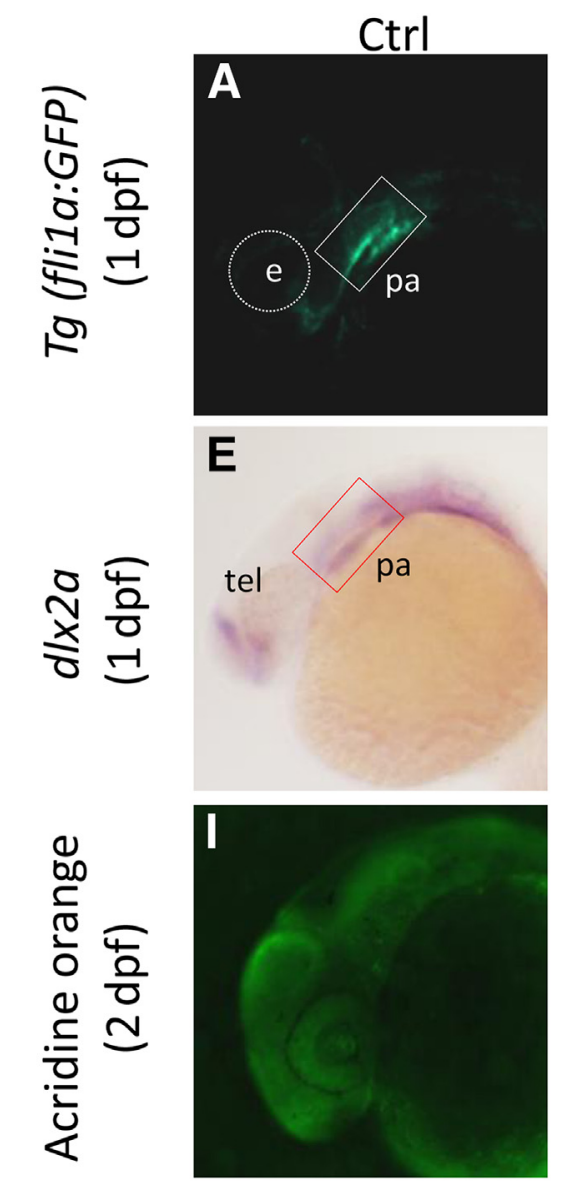

M

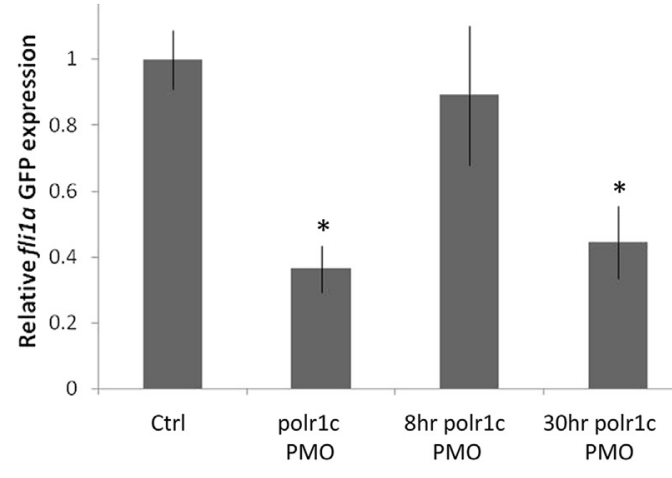

0

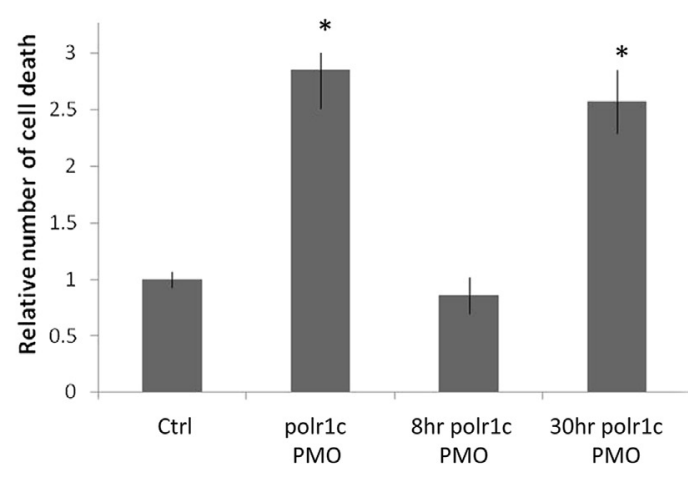

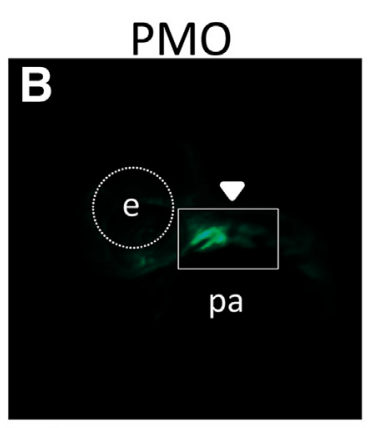

$\mathbf{F}$
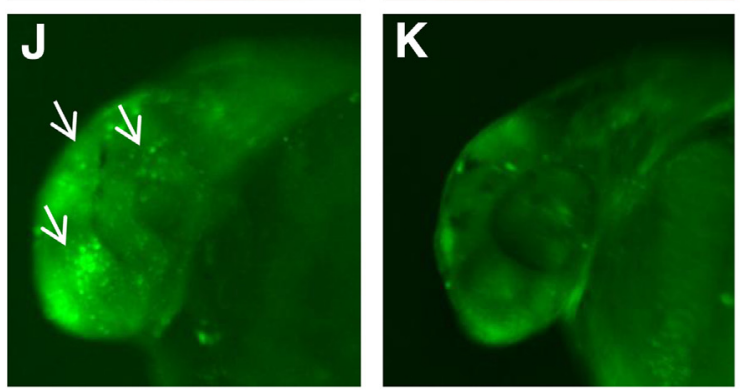

$\mathbf{N}$

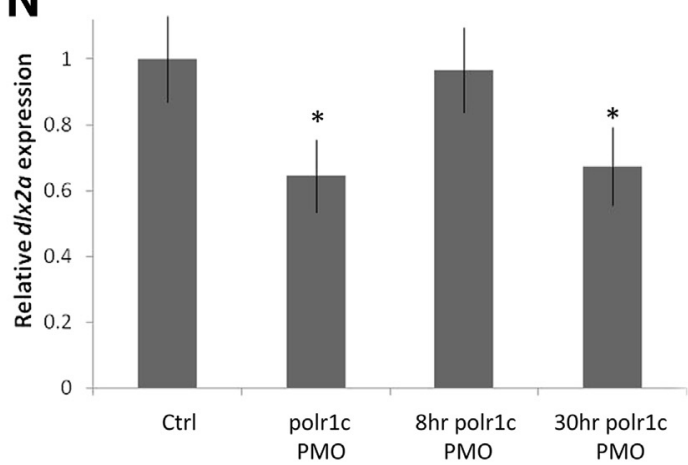

Figure 2 The restoration of polr $1 \mathrm{c}$ at $8 \mathrm{hpf}$ can correct the neural crest cell (NCC) specification and reduce cell death in the P-morphants. A-D: polr1c PM0 was injected into the transgenic line Tg(fli1a:GFP), in which the ectomesenchyme was labeled. fli1a expression at $1 \mathrm{dpf}$ is largely reduced in the pharyngeal arch in the polr1c P-morphants (B; arrowhead) and P-morphants that were exposed to UV light at 30 hours (D; arrowhead). $\mathbf{E}-\mathbf{H}$ : Decreased $d l \times 2 a$ expression in the pharyngeal arch region (red arrows) in 1-dpf polr1c P-morphants (F) and P-morphants that were exposed to UV light at 30 hours $(\mathbf{H})$ when compared to controls (E) and P-morphants that were exposed to $365-\mathrm{nm}$ UV light at $8 \mathrm{hpf}(\mathbf{G})$. I-L: Acridine orange staining shows increased cell death (white arrows) in both polr1c P-morphants (J) and P-morphants that were exposed to UV light at $30 \mathrm{hpf}$ (L) when compared to controls (I) and P-morphants that were exposed to UV light at $8 \mathrm{hpf}(\mathbf{K})$. Anterior is to the left. M-0: Quantified data of panels A-L. M: fli1a expression in 1-dpf embryos. The signal was measured within the white boxes. N: $d l x 2 a$ expression in 1-dpf embryos. The signal was measured within the red boxes. 0: Relative fluorescent signal of cell death was measured in the 2-dpf embryos. $n=25$ (M-0). ${ }^{*} P<0.05$ versus control. Scale bars $=100 \mu \mathrm{m}$. e, eye; pa, pharyngeal arches; tel, telencephalon. 

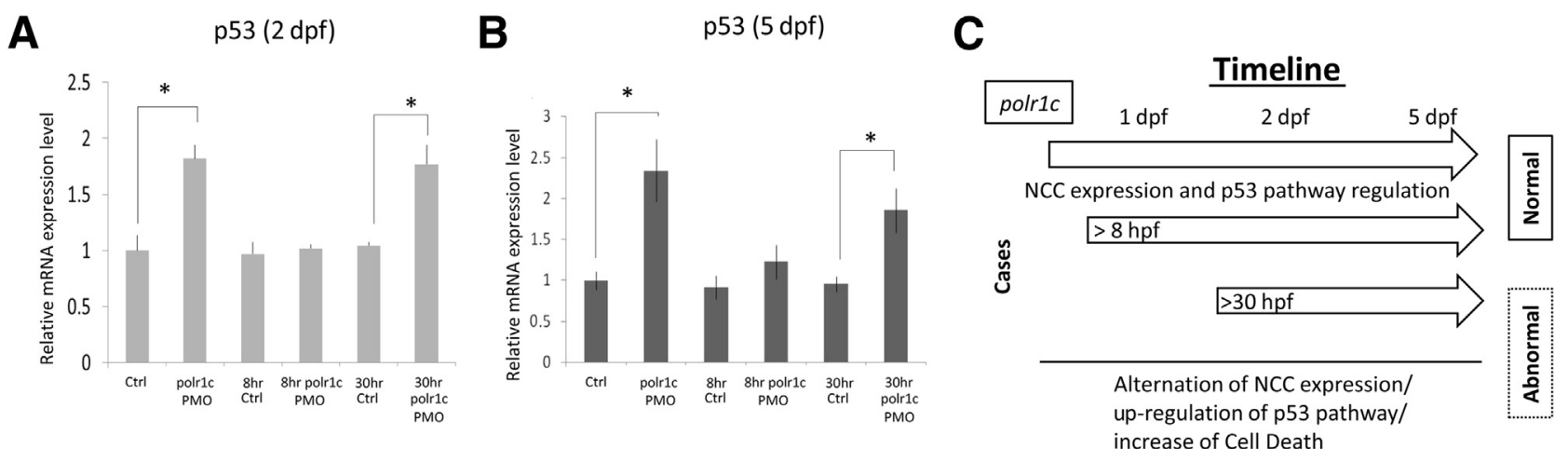

Figure 3 The restoration of polr1c at $8 \mathrm{hpf}$ can normalize the $p 53$ pathway in the P-morphants. A and B: Real-time quantitative PCR shows that the polr1c P-morphants and P-morphants that were exposed to UV light at $30 \mathrm{hpf}$ have a higher mRNA expression level of $p 53$ expression at $2 \mathrm{dpf}(\mathbf{A})$ and $5 \mathrm{dpf}$ (B); whereas the P-morphants exposed to $365-\mathrm{nm}$ UV light at $8 \mathrm{hpf}$ have a similar $p 53$ expression level as controls. C: Summary of the study. In the wild-type case (top), polr1c is expressed throughout the development, and normal facial structure is formed. In the morphant case (bottom), polr1c is not expressed during the development, and thus results in facial malformation, which is due to the missexpression of NCCs, increase of cell death, and activation of $p 53$ pathway. In the case of P-morphants exposed to UV light, the facial malformation phenotype can be recovered if polr1c is restored at 8 hpf; however, the anomalies cannot be solved if polr1c restoration is done after $30 \mathrm{hpf}$. The restoration of polr1c at an earlier developmental stage can rescue the facial phenotype via correcting the NCC expression and $p 53$ pathway. Data are expressed as means \pm SEM. $n=12$ (A and $\mathbf{B})$. ${ }^{*} P<0.05$. Ctrl, control.

malformation of the polrlc mutant at $5 \mathrm{dpf}$, which the photomorphants (P-morphants) exhibited a rounded head, smaller eyes, and jaw agenesis at $5 \mathrm{dpf}$ (Figure 1, A-D). The facial phenotypes were further confirmed by Alcian blue staining (Figure 1, E-H). The P-morphant displayed a shorter and smaller ethmoid plate structure (Figure 1, I-L). The result can be clearly seen in the enlarged ethmoid plate (Figure 1, $\mathrm{M}-\mathrm{P})$. Different degrees of cranioskeletal anomalies were found in the morphants, and the result was quantified by various parameters of the ethmoid plate. Briefly, the horizontal length, longitudinal width, and the relative area of the ethmoid plate were measured. Results clearly showed that the length, width, and relative area in P-morphants and the 30hours UV light-exposed P-morphants were significantly smaller than those in the controls. However, the 8-hours UV light-exposed morphants exhibited a similar structure as controls. In addition, the length to width ratio of the ethmoid plates was similar among controls and P-morphants (Figure 1, $\mathrm{Q}-\mathrm{T})$.

\section{Restoration of polr1c Plays Regulatory Role in NCC Expression, Cell Death Mechanism, and p53 Pathway}

The expression pattern of the NCCs was monitored by the use of transgenic fish and in situ hybridization. The $\operatorname{Tg}(f l i l a: G F P)$ line was used to monitor the pharyngeal arches development. flila is a marker of neural crest-derived ectomesenchyme, ${ }^{20}$ whose expression was severely reduced in P-morphants when compared to controls and 8-hpf P-morphants (Figure 2, A-D and M). A similar result was found in the other early cranial NCC marker $d l x 2 a$. Its expression at pharyngeal arches was diminished at $1 \mathrm{dpf}$ in the P-morphants (Figure 2, E-H and N). These results clearly demonstrated that the restoration of polrlc in early embryogenesis ( $8 \mathrm{hpf}$ ) may bring back the normal
NCC specification at $1 \mathrm{dpf}$, which is required for normal facial development.

In addition, extensive cell death in early embryogenesis was found in the TCS morphant and mutant. ${ }^{10}$ As expected, the P-morphants and P-morphants exposed to UV light at 30 hpf showed extensive cell death when compared to the controls at 2 dpf. However, the P-morphants that were exposed to the UV light at $8 \mathrm{hpf}$ displayed reduced cell death phenotype (Figure 2, I-L and O). Moreover, the activation of p53 expression in TCS was suppressed in the P-morphants exposed to the UV light at $8 \mathrm{hpf}$ (Figure 3, A and B). The activation of the $p 53$ pathway leads to cell cycle arrest and apoptosis, ${ }^{21}$ together with the altered NCC expression, resulted in the facial malformation in the TCS. ${ }^{22}$ Taken together, these results suggested that polrlc contributes to facial development during early embryogenesis in a critical time period in which polrlc contributes to regulation of NCC specification and function of the $p 53$ pathway (Figure 3C).

\section{Discussion}

Zebrafish have been recruited as a new human disease model, ${ }^{23,24}$ in which they share similar developmental pathways and signaling pathways as humans. In terms of craniofacial development, there are a number of regulatory developmental mechanisms shared between fish and mammals. $^{25,26}$

Facial development requires proper cranial NCC specification and migration. The facial malformation in TCS was found to be closely related to NCC expression. ${ }^{8,27-30}$ Correct migration of NCC is critical for palatogenesis. ${ }^{9}$ One of the major causes of the facial malformation phenotype in Type 3 TCS was due to the improper NCC localization. ${ }^{10,22,31}$ It is crucial for correct NCC migration during the early developmental stage. Our data showed that the 
reactivation of polrlc at early embryogenesis can partially rescue the facial phenotype, which was due to the recovery of NCC expression. The study by Noack Watt and colleagues $^{31}$ suggested that the excessive cell death in polrlc mutants diminished the pool of pre-migratory NCCs, which then led to reduced migrating NCCs, and resulted in facial malformation. Our result here showed that the recovery of the facial structure may only be achieved by the early restoration of polrlc expression, which further supported the notion of the regulatory roles of polrlc in the early stage of facial formation. However, it should be noted that the recent study used solely the ethmoid plate as the parameter for the facial malformation. Furthermore, the rescued morphants could not totally recover the facial structure, such as the rostral edge of the ethmoid plate. Such phenotype can be classified as "cleft palate." ${ }^{32,33}$ As with other rescue experiments, $100 \%$ recovery was not found in zebrafish or mouse models. ${ }^{9,10,31,34}$ Nevertheless, our study showed the restoration of polrlc at early developmental stage may improve the ethmoid plate structure significantly. It should be noted that various facial phenotypes can be found in TCS patients. In the zebrafish model, the underdevelopment of the lower jaw and trabecula of the neurocranium in the morphants from the whole-mount Alcian blue staining could be seen. Various signaling pathways are tightly regulated to form the facial structure, and our group is now undergoing different experiments to understand the regulatory roles of polrlc in the facial formation.

Studies have found that the $p 53$ suppression can rescue the TCS phenotype in mouse and zebrafish models. ${ }^{9,10,31}$ However, the long-term suppression of $p 53$ may result in a higher risk of tumors during their life time..$^{30,35,36}$ Thus, alternative therapeutic treatment, such as the use of antioxidants, has been suggested recently. ${ }^{34}$ Because possible off-target effects may be generated via the treatment, limiting the treatment by designed time period would reduce the possible side effects. We here suggest that polrlc may not be critical for facial development after $30 \mathrm{hpf}$, and thus propose the possibility of withdrawing the chemotherapies after that time point. Although such withdrawal is not easy in in utero mammalian models, the identification of the polrlc functional time window in this study should provide some insights on potential treatment scheme.

\section{Conclusion}

The restoration of polrlc at early embryogenesis can partially rescue the TCS facial malformation phenotype via correcting the NCC specification and normalizing the $p 53$ mRNA expression level.

\section{Supplemental Data}

Supplemental material for this article can be found at https://doi.org/10.1016/j.ajpath.2017.10.004.

\section{References}

1. The Treacher Collins Syndrome Collaborative Group, Dixon J, Edwards SJ, Gladwin AJ, Dixon MJ, Loftus SK, Bonner CA, Koprivnikar K, Wasmuth JJ: Positional cloning of a gene involved in the pathogenesis of Treacher Collins syndrome. Nat Genet 1996, 12: 130-136

2. Collins ET: Case with symmetrical congenital notches in the outer part of each lower lid and defective development of the malar bones. Trans Ophthalmol Soc U K 1900, 20:190

3. Su PH, Chen JY, Chen SJ, Yu JS: Treacher Collins syndrome with a de Novo 5-bp deletion in the TCOF1 gene. J Formos Med Assoc 2006, 105:518-521

4. Kadakia S, Helman SN, Badhey AK, Saman M, Ducic Y: Treacher Collins Syndrome: the genetics of a craniofacial disease. Int J Pediatr Otorhinolaryngol 2014, 78:893-898

5. Plomp RG, van Lieshout MJ, Joosten KF, Wolvius EB, van der Schroeff MP, Versnel SL, Poublon RM, Mathijssen IM: Treacher Collins syndrome: a systematic review of evidence-based treatment and recommendations. Plast Reconstr Surg 2016, 137:191-204

6. Dauwerse JG, Dixon J, Seland S, Ruivenkamp CA, van Haeringen A, Hoefsloot LH, Peters DJ, Boers AC, Daumer-Haas C, Maiwald R, Zweier C, Kerr B, Cobo AM, Toral JF, Hoogeboom AJ, Lohmann DR, Hehr U, Dixon MJ, Breuning MH, Wieczorek D: Mutations in genes encoding subunits of RNA polymerases I and III cause Treacher Collins syndrome. Nat Genet 2011, 43:20-22

7. Vincent M, Genevieve D, Ostertag A, Marlin S, Lacombe D, MartinCoignard D, et al: Treacher Collins syndrome: a clinical and molecular study based on a large series of patients. Genet Med 2016, 18:49-56

8. Dixon J, Jones NC, Sandell LL, Jayasinghe SM, Crane J, Rey JP, Dixon MJ, Trainor PA: Tcof1/Treacle is required for neural crest cell formation and proliferation deficiencies that cause craniofacial abnormalities. Proc Natl Acad Sci U S A 2006, 103:13403-13408

9. Jones NC, Lynn ML, Gaudenz K, Sakai D, Aoto K, Rey JP, Glynn EF, Ellington L, Du C, Dixon J, Dixon MJ, Trainor PA: Prevention of the neurocristopathy Treacher Collins syndrome through inhibition of p53 function. Nat Med 2008, 14:125-133

10. Lau MC, Kwong EM, Lai KP, Li JW, Ho JC, Chan TF, Wong CK, Jiang YJ, Tse WK: Pathogenesis of POLR1C-dependent Type 3 Treacher Collins syndrome revealed by a zebrafish model. Biochim Biophys Acta 2016, 1862:1147-1158

11. Kimmel CB, Ballard WW, Kimmel SR, Ullmann B, Schilling TF: Stages of embryonic development of the zebrafish. Dev Dyn 1995, 203:253-310

12. Tse WKF, Eisenhaber B, Ho SHK, Ng Q, Eisenhaber F, Jiang YJ: Genome-wide loss-of-function analysis of deubiquitylating enzymes for zebrafish development. BMC Genomics 2009, 10:637

13. Pfaffl MW: A new mathematical model for relative quantification in real-time RT-PCR. Nucleic Acids Res 2001, 29:e45

14. Solomon KS, Kudoh T, Dawid IB, Fritz A: Zebrafish foxi1 mediates otic placode formation and jaw development. Development 2003, 130: 929-940

15. Tse WK, Jiang YJ: Functional screen of zebrafish deubiquitylating enzymes by morpholino knockdown and in situ hybridization. Methods Mol Biol 2012, 815:321-331

16. Fong HC, Ho JC, Cheung AH, Lai KP, Tse WK: Developmental toxicity of the common UV filter, benophenone-2, in zebrafish embryos. Chemosphere 2016, 164:413-420

17. Tallafuss A, Gibson D, Morcos P, Li Y, Seredick S, Eisen J, Washbourne P: Turning gene function $\mathrm{ON}$ and $\mathrm{OFF}$ using sense and antisense photo-morpholinos in zebrafish. Development 2012, 139: 1691-1699

18. Saxena A, Peng BN, Bronner ME: Sox 10-dependent neural crest origin of olfactory microvillous neurons in zebrafish. Elife 2013, 2:e00336

19. Deng W, Farnham MM, Goldys EM, Mohammed S, Pilowsky PM: Gene interference with morpholinos in a gold nanoparticle-based 
delivery platform in rat PC12 cells. J Biomed Nanotechnol 2015, 11 : $2111-2123$

20. Das A, Crump JG: Bmps and id2a act upstream of Twist1 to restrict ectomesenchyme potential of the cranial neural crest. PLoS Genet 2012, 8:e1002710

21. Rubbi CP, Milner J: Disruption of the nucleolus mediates stabilization of p53 in response to DNA damage and other stresses. EMBO J 2003, 22:6068-6077

22. Tse WK: Treacher Collins syndrome: new insights from animal models. Int J Biochem Cell Biol 2016, 81:44-47

23. Zon LI, Peterson RT: In vivo drug discovery in the zebrafish. Nat Rev Drug Discov 2005, 4:35-44

24. Seth A, Stemple DL, Barroso I: The emerging use of zebrafish to model metabolic disease. Dis Model Mech 2013, 6:1080-1088

25. Swartz ME, Sheehan-Rooney K, Dixon MJ, Eberhart JK: Examination of a palatogenic gene program in zebrafish. Dev Dyn 2011, 240: 2204-2220

26. Yelick PC, Schilling TF: Molecular dissection of craniofacial development using zebrafish. Crit Rev Oral Biol Med 2002, 13:308-322

27. Poswillo D: The pathogenesis of the Treacher Collins syndrome (mandibulofacial dysostosis). Br J Oral Surg 1975, 13:1-26

28. Wiley MJ, Cauwenbergs P, Taylor IM: Effects of retinoic acid on the development of the facial skeleton in hamsters: early changes involving cranial neural crest cells. Acta Anat (Basel) 1983, 116: 180-192
29. Herring SW, Rowlatt UF, Pruzansky S: Anatomical abnormalities in mandibulofacial dysostosis. Am J Med Genet 1979, 3:225-229

30. McKeown SJ, Bronner-Fraser M: Saving face: rescuing a craniofacial birth defect. Nat Med 2008, 14:115-116

31. Noack Watt KE, Achilleos A, Neben CL, Merrill AE, Trainor PA: The roles of RNA polymerase I and III subunits Polr1c and Polr1d in craniofacial development and in zebrafish models of Treacher Collins syndrome. PLoS Genet 2016, 12:e1006187

32. Kamel G, Hoyos T, Rochard L, Dougherty M, Kong Y, Tse W, Shubinets V, Grimaldi M, Liao EC: Requirement for frzb and fzd7a in cranial neural crest convergence and extension mechanisms during zebrafish palate and jaw morphogenesis. Dev Biol 2013, 381:423-433

33. Eberhart JK, He X, Swartz ME, Yan YL, Song H, Boling TC, Kunerth AK, Walker MB, Kimmel CB, Postlethwait JH: MicroRNA Mirn140 modulates Pdgf signaling during palatogenesis. Nat Genet 2008, 40:290-298

34. Sakai D, Dixon J, Achilleos A, Dixon M, Trainor PA: Prevention of Treacher Collins syndrome craniofacial anomalies in mouse models via maternal antioxidant supplementation. Nat Commun 2016, 7:10328

35. Donehower LA, Harvey M, Slagle BL, McArthur MJ, Montgomery CA Jr, Butel JS, Bradley A: Mice deficient for p53 are developmentally normal but susceptible to spontaneous tumours. Nature 1992, 356:215-221

36. Levine AJ: p53, the cellular gatekeeper for growth and division. Cell 1997, 88:323-331 